\title{
Computer Simulation of the Mechanism for the Synthesis of Carbonado Diamond
}

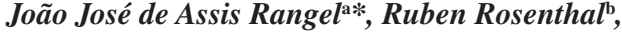 \\ Guerold Sergueevitch Bobrovnitchii ${ }^{\mathrm{b}}$, Sergio Neves Monteiro ${ }^{\mathrm{b}}$ \\ ${ }^{a}$ Candido Mendes University, UCAM, Campos, NPDI, Rua Anita Pessanha, 100, \\ 28040-320 Campos dos Goytacazes - RJ, Brazil \\ ${ }^{\mathrm{b}}$ Laboratory for Advanced Materials - LAMAV, \\ State University of the Northern Rio de Janeiro - UENF, \\ Av. Alberto Lamego 2000, 28015-620 Campos dos Goytacazes - RJ, Brazil
}

Received: June 28, 2006; Revised: December 8, 2006

\begin{abstract}
The synthesis mechanism associated with the formation of carbonado type polycrystalline diamond has been analyzed. This analysis was performed by computer simulation of the field of temperature developed at the moment of graphite transformation into polycrystalline diamond. It was found that during the synthesis of this carbonado diamond, a strong non-steady thermal condition occurs, leading to changes in the thermodynamic equilibrium. As a consequence, a region comprising non-transformed graphite and carbonado with different characteristics is established. An experimental comparison was carried out between the carbonado hardness profile and the field of temperatures. The results show that the higher the temperature attained at local points inside the high-pressure chamber, the higher the hardness of the diamond. The simulation and experimental results indicated that concurrent mechanisms are acting during the carbonado synthesis.
\end{abstract}

Keywords: computer simulation, carbonado, polycrystalline diamond, field of temperature

\section{Introduction}

Diamond is known as the material with highest hardness in addition to other special properties such as very high thermal conductivity and electric resistivity ${ }^{1}$. Owing to characteristics related to size, morphology and inclusions, as well as micro structural defects, such as flaws and grain boundaries, different types of diamond are recognized for both, the naturally occurring and the synthetically produced types ${ }^{2}$. For gemstone quality, natural large and flawless single crystals with specific lapidated morphology are of paramount importance. However, for industrial applications such as in drilling, cutting and polishing, the strength and hardness are the basic requirements. For this reason most synthetic diamonds are produced with a smaller size, in the form of dust or powder, since the strength decreases with increasing crystalline size ${ }^{3}$.

Polycrystalline diamonds may not be of interest for jewellery, due to grain boundaries that impair the transparency, but they do present practical advantages regarding the above-mentioned industrial applications. In nature, polycrystalline diamonds are found mostly in Brazil and South Africa with specific denominations such as framesite, ballas and carbonado ${ }^{4}$. Carbonado type synthetic polycrystalline diamond (CSPD) is also produced at high pressure and high temperature (HPHT) conditions in the presence of a metallic alloy molten catalyst ${ }^{5}$. Some relevant differences apparently exist between the SCPD and the conventional crystalline synthetic diamonds. Metallic inclusions with totaling up to $10 \%$ in weight tend to segregate to the grain boundaries of the SCPD, which reduces its thermal stability. Moreover stronger non-equilibrium conditions are also believed to be responsible for the carbonado structure formation ${ }^{6}$.

The graphite to diamond, $\mathrm{G} \rightarrow \mathrm{D}$, transformation mechanism is a very peculiar one for the CSPD. While conventional crystalline synthetic diamonds are nucleated from carbon dissolved in the molten alloy, the CSPD results in a bulk martensitic transformation of the graphite $^{7}$. As the catalyst alloy melts, the high pressure applied to the system permits metallic liquid penetration through the graphite pores.
This promotes the $\mathrm{G} \rightarrow \mathrm{CSPD}$ by means of a solid-state martensitic reaction, which takes place within the penetrated graphite volume. For this reaction to occur, the HPHT conditions must be away from the line of graphite/diamond thermodynamic equilibrium to allow a higher Gibbs free energy ${ }^{8}$. The P, T and t parameters at which this phenomenon has been observed are: 6.5 to $8.5 \mathrm{GPa}, 1500$ to $1800{ }^{\circ} \mathrm{C}$ for processing times of the order of 5 to 10 seconds $^{9,10}$.

In the present work a mathematical model based on the finite difference technique was created to allow a computer simulation of the temperature distribution during transformation of a CSPD. In the model, the temperature is determined by means of the heat generated by the flow of an electric current established inside the High Pressure Reaction Chamber (HPRC). The simulation results permitted the determination of the field of temperatures (FT) associated with all temperatures inside the HPRC at the moment of the G $\rightarrow$ CSPD transformation. Actually, the FT is associated with the temperature distribution occurring during the synthesis process. This is entirely different from the profile of temperatures derived by common models that consider any given instant of the process under equilibrium conditions ${ }^{11,12}$.

One specific advantage of the model is that the influence on the heat generated by the electric current in the different component materials, including the transformed diamond, is taken into account. The model also considers the exothermic effect resulting from the $\mathrm{G} \rightarrow$ CSPD phase transformation as well as the penetration of molten metal in the graphite pores.

\section{Mapping of Temperatures}

Figure 1 shows a schematic section of the toroidal HPRC, which was considered for the computer determination of the temperature distribution. Owing to the symmetry of the chamber, only its left half is shown in Figure 1. 


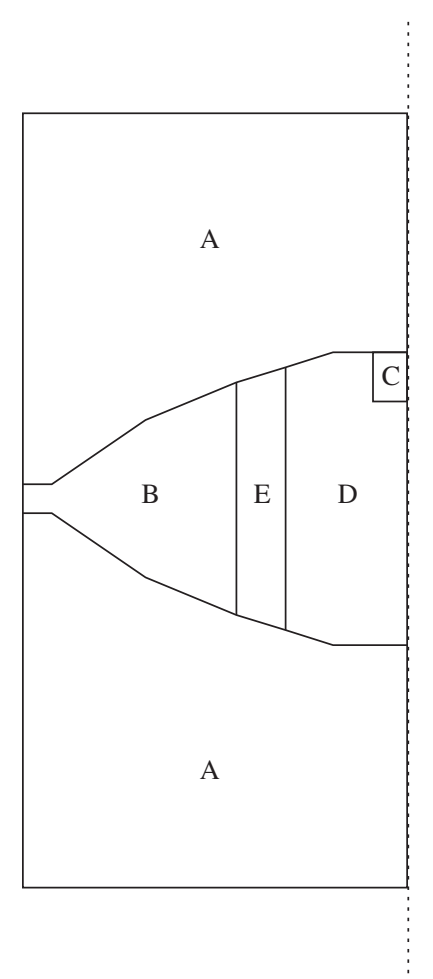

Figure 1. High-pressure chamber. (A- matrix, B-gasket, C-catalyst metal, D- graphite reactive cell and $\mathbf{E}$ - graphite heating sleeve). Due to the cylindrical symmetry, only the left part of the chamber is shown.

For the evaluation of the temperature distribution, the computer simulation considered the change in the applied voltage for the heating process inside the HPRC and a graphite pore radius $\mathrm{R}=10^{-5} \mathrm{~mm}$.

Other parameters selected for the simulation were a $\mathrm{G} \rightarrow \mathrm{CSPD}$ transformation yield (percentage of converted carbonado) equal to $70 \%$ and a processing time of 5 seconds, which corresponds to a common industrial synthesis operation ${ }^{5}$.

Figure 2 shows one simulation result presenting the temperature distribution in the reactive cell, (sector D in Figure 1) where the $\mathrm{G} \rightarrow \mathrm{CSPD}$ transformation occurred. As shown in Figure 2, the body of the transformed CSPD, represented by the upper gray shaded area, occupies about $70 \%$ of the cell. Temperature values determined by the simulation are displayed in tones of gray; the higher the temperature the darker the tone. The temperature distribution in Figure 2, suggests the existence of three distinct regions at the moment of the phase transformation. Region (1), in the core of the reactive cell, with an approximately spherical shape, corresponds to the interior of the transformed CSPD. Temperatures in this region (1) are relatively lower and vary from $1400{ }^{\circ} \mathrm{C}$ to $1600{ }^{\circ} \mathrm{C}$. Region (2), in darker tones, concentric with the first region and corresponding to a middle zone of the CSPD. This region is associated with higher temperatures, $1800{ }^{\circ} \mathrm{C}$ to $2000{ }^{\circ} \mathrm{C}$. Region (3), in lighter gray tones, also concentric with the other two regions, corresponding to the external zone of the CSPD formed at lower temperatures, $1600{ }^{\circ} \mathrm{C}$ to $1400{ }^{\circ} \mathrm{C}$, which are comparable with those in Region (1).

The results shown in Figure 2 indicate that different zones of the CSPD body are formed at different temperatures or thermodynamic conditions. As a consequence, different morphological structures and properties could be expected at different parts of the CSPD body. This result was also found by Ekimov et al. ${ }^{6}$ investigating the synthesis of carbonado-like polycrystalline diamond in the $\mathrm{B}_{4} \mathrm{C}$-graphite system.

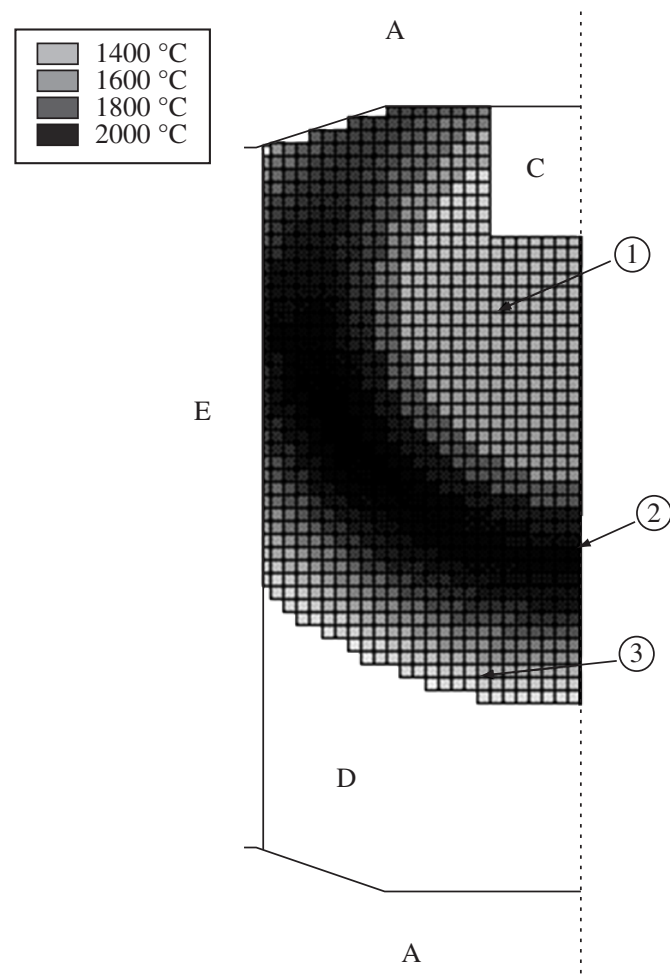

Figure 2. Field of temperatures associated with the formation of CSPD from graphite. Applied voltage $1.8 \mathrm{~V}$ and processing time 5 seconds.

By comparing the profiles of transformation temperatures Semionova-Tian-Chansckaia and Ovchinnikov ${ }^{11}$ found a direct association with the corresponding diamond hardness. The part of the diamond body formed at higher temperatures, region (2) in Figure 2, displays correspondently higher hardness values, whereas diamond parts with lower hardness, regions (1) and (3) are associated with lower temperatures.

\section{Analysis of the Temperature Variation in the Synthesis of CSPD}

As already mentioned, the heating during the synthesis process is achieved using electricity. However, during the $\mathrm{G} \rightarrow \mathrm{CSPD}$ transformation an exothermic effect also occurs, which generates additional heat for the process. Therefore, two simultaneously operating heat sources promote a significant variation in the reactive zone temperatures, which results in the FT profile shown in Figure 2. As a consequence, the variation in temperature at the moment of transformation may reach $600{ }^{\circ} \mathrm{C}$, under proper thermodynamic conditions.

Figure 3 shows the pressure-temperature equilibrium diagram for carbon, limited to conditions of industrial interest for $\mathrm{G} \rightarrow$ CSPD transformation. Points 1 and 2 in Figure 3 correspond to the thermodynamic parameters associated with the three distinct CSPD regions identified in Figure 2. The significant variation in temperature that exists in region (2) as compared with (1) and (3) may be explained as follows.

At the beginning of the process, after the appropriate pressure has been applied, the temperature in the system is raised to point 1 by simple electrical heating. Point 1 is not only above the equilibrium line, Figure 3, corresponding to diamond stability, but also above the melting temperature for the catalyst metal. Consequently molten metal begins to penetrate the graphite pores and to promote the bulk 


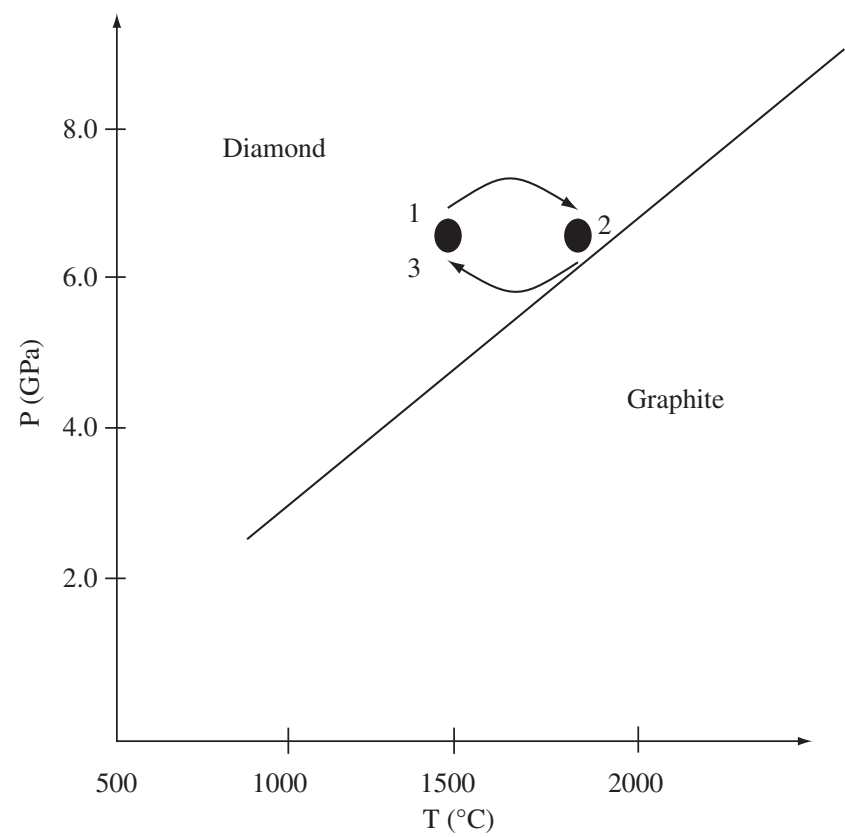

Figure 3. $\mathrm{P}$ and $\mathrm{T}$ equilibrium diagram for carbon indicating conditions for the different regions of the CSPD (pressure of $6.5 \pm 0.1 \mathrm{GPa}$ and temperature range from $1500{ }^{\circ} \mathrm{C}$ to $1800 \pm 50{ }^{\circ} \mathrm{C}$ ).

$\mathrm{G} \rightarrow$ CSPD transformation ${ }^{5}$. As this transformation occurs, exothermic heat from the phase reaction itself is added to the electrical heat, and the temperature therefore increases to that at point 2 .

This continuous formation of CSPD at an increasing temperature corresponds to the evolution from region (1) to (2) in Figure 2, which is associated with a diamond body being synthesized from $1400{ }^{\circ} \mathrm{C}$ to $2000{ }^{\circ} \mathrm{C}$. By the time point 2 is reached, $50 \%$ of CSPD is already transformed. The replacement of half of the conducting graphite in the chamber by the insulating diamond body poses a limitation to the electrical current. The heat generated is decreased and the temperature drops to that corresponding to point 1 , in Figure 3 . The process is then completed and another layer with $20 \%$ of diamond, region (3) in Figure 2, is incorporated to the CSPD body.

\section{Analysis of the Formation Mechanism of the CSPD}

In order to investigate the practical consequences of the field of temperature predicted by computer simulation, several CSPD samples were experimentally produced in a toroidal anvil high-pressure chamber with a $13.5 \mathrm{~mm}$ central concavity. The pressure of $6.5 \pm 0.1 \mathrm{GPa}$ was provided by a 1000 ton model D0040 RYAZANTIASMASS Russian press equipment. The operational FT was established after applying $1.8 \mathrm{~V}$ for 5 seconds. The experimental values of temperature were determined with a precision of $\pm 50{ }^{\circ} \mathrm{C}$.

One of the CSPD samples was longitudinally ruptured to allow a direct comparison with the simulated section shown in Figure 2. The ruptured sample surface was etched with nitric acid to dissolve the local metallic phase characteristic of the CSPD structure. This surface was then metallized with gold and observed by scanning electron microscopy, SEM, in a model 962 Zeiss equipment. Examples of the typical fracture structure observed for each region of the FT are now presented.

Figure 4 illustrates the fractured surface observed by SEM at the two typical regions corresponding to the schematic longitudinal section of the CSPD in Figure 2. It should be noticed that Figure 4b, associated with region (2), closer to the equilibrium line (Figure 3), displays a more faceted structure with evidence of cubic crystals. By contrast, Figure 4a, region (1), which is relatively far from the equilibrium line, show a rougher structure with fewer facets and crystal-like elements. Some plate or layer features are also shown in Figure 4c.

The diamond crystal shown in Figure $4 \mathrm{~b}$ displays similar morphological characteristics as those synthesized in $\mathrm{B}_{4} \mathrm{C}-\mathrm{C}$ system by Ekimov et al. ${ }^{6}$.

\section{Discussion}

The results obtained in the present work regarding the influence of the field of temperatures FT developed inside a high-pressure reaction chamber, HPRC, on the formation of carbonado diamonds, permitted the explanation of the non-uniform distribution of hardness.

The existence of a hardness profile in the CSPD was reported by Semionova-Tian-Chansckaia and Ovchinnikov ${ }^{13}$, however they did not present a clear explanation for this feature. Actually they simply proposed that a possible temperature gradient inside the HPRC could be responsible for the different values of hardness found throughout the CSPD.

The computer simulation carried out in the present work provided evidence that the non-uniform distribution of hardness is not a consequence of a simple temperature gradient, as suggested ${ }^{13}$. As a matter of fact, the FT obtained by the present computer modeling is not in agreement with a continuous gradient.

As shown in Figure 2, one can consider the existence of two distinct regions in the CSPD due to its formation involving different thermodynamic conditions. This was verified in terms of the corresponding transformation temperatures, given by the computer model for the FT, for each of the three regions. It was actually found that two regions, (1) and (3), were formed at the same interval of temperatures, 1400 to $1600{ }^{\circ} \mathrm{C}$. These two regions are physically separated, Figure 2, by another region (2), formed at higher temperatures of 1800 to $2000{ }^{\circ} \mathrm{C}$. Consequently, the external parts of the CSPD body are made of diamond grains formed at lower temperatures whereas its interior is made of higher temperature diamond grains.

The morphological analysis revealed that the lower temperature regions, Figure $4 \mathrm{a}-\mathrm{c}$, present the typical CSPD rough and layered structure characteristic of the graphite from which it was formed. On the contrary, the intermediate region (2) displayed facetted and crystal structures that are not typical of a CSPD. In fact, these features, some with sizes up to $40 \mu \mathrm{m}$, are more like to be found in single crystalline diamonds.

The association between the values of microhardness obtained in a CSPD cross section by Seminova-Tian-Chansckaia and Ovchinnikov ${ }^{13}$ and the temperatures at the moment of its formation, Figure 2, has shown that regions (1) and (3) have lower microhardness as compared to region (2). In other words, that part of the CSPD transformed at higher temperatures presents relatively higher values of microhardness. It is then possible to infer that the non-uniform distribution of microhardness is a consequence of the different characteristics developed in distinct parts of CSPD body due to the FT. For instance, region (2) with apparently more perfect diamond crystals, Figure 4b, presents a comparatively higher microhardness.

Combined with these experimental results, the computer model for the FT explains the non-uniform values of microhardness throughout the CSPD. In principle, by controlling the temperature distribution inside the HPRC, the model could be used to improve the mechanical strength and to make the microhardness more uniform.

Another point revealed by the experimental results in the present work is a rather complex transformation associated with the CSPD. 


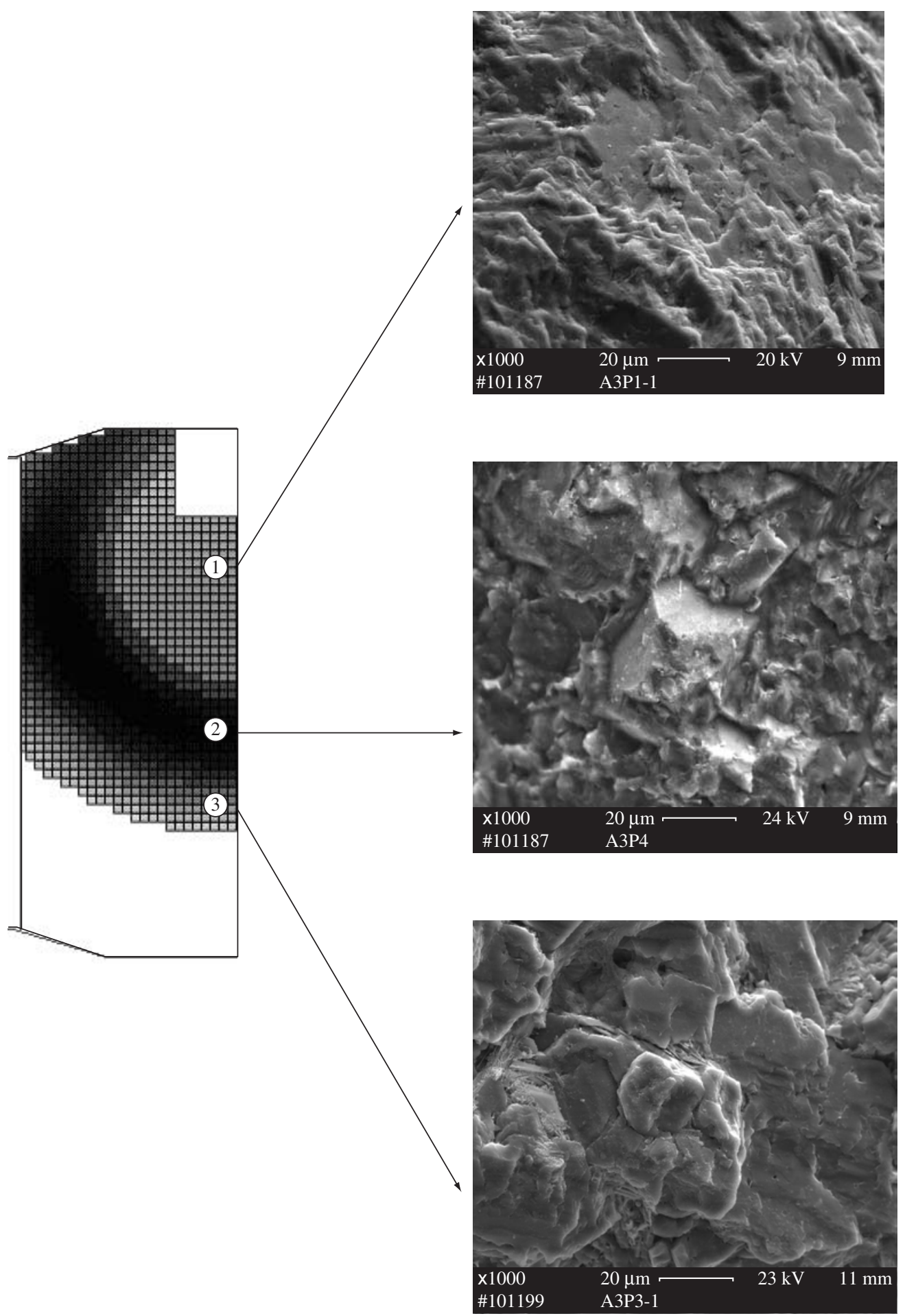

Figure 4. Typical SEM micrographic aspects of: a) region (1); b) region (2); c) region (3) of the CSPD.

The fact that single crystalline diamonds with relatively larger sizes $(40 \mu \mathrm{m})$ were found in the middle of the CSPD body, indicates a possible mechanism other than that of the martensitic transformation. Since the literature on CSPD is restricted to a martensitic mechanism ${ }^{5}$, the experimental results of this work display a complex situation, which is worth discussing.

As shown in Figure 3, the process parameters related to the intermediate region (2) are closer to the $\mathrm{G} \leftrightarrow \mathrm{D}$ thermodynamic equilibrium line. In practice, graphite under $\mathrm{P}$ and $\mathrm{T}$ conditions near the equilibrium line in the carbon phase diagram usually transform into single crystalline diamond $d^{5,7}$. In this case, the transformation involves diffusion assisted nucleation of diamond by carbon solution in the molten catalyst alloy, which is different from the martensite assisted CSPD transformation ${ }^{9,10}$. Therefore, besides the non-uniform microhardness distribution, the present work has shown evidence of non-uniform CSPD morphology probably associated with different transformation mechanisms. 


\section{Conclusions}

A computer simulation of the field of temperature developed at the moment of graphite transformation into carbonado type synthetic polycrystalline diamond, inside an anvil with central concavity high pressure reaction chamber, has displayed non-uniform characteristics.

The reason for this complex non-uniformity is caused by the FT developed at the moment of transformation. This results in different transformation mechanisms that affect the morphology and influence the mechanical properties.

The general analysis of the results obtained in the present work has displayed, for the first time, the possible existence of concurrent mechanisms during the CSPD synthesis. This motivates further discussions concerning the theories presented so far to explain the formation of this type of diamond.

\section{Acknowledgments}

The authors thank the support for this investigation provided by the Brazilian agencies; CNPq, CAPES, FAPERJ and FENORTE. Special thanks are due to Dr. Andrei Pontemkin for supplying the diamond samples.

\section{References}

1. Howes VR. Physical Properties of Diamonds. ed. R. Berman, Clarendon Press, Oxford, United Kingdom; 1965. p. 174-183.

2. Field JE. The Properties of Diamond, Academic Press, London, United Kingdom; 1979. 281p.

3. Skury ALD, Bobrovnitchii GS, Monteiro SN, A Parametric Relationship for Synthesized Diamond Powder. Diamond and Related Materials. 2006; 15(1):61-66
4. Jeynes C. Natural polycrystalline diamond. Industrial Diamond Review. 1978; 1:14-23.

5. Poliakov VP, Nozhkina AV, Chirikov NV. Diamonds and Superhard Materials, ed. 1, Metallurgy (in Russian), Moscow; 1990. 325p.

6. Ekimov EA, Sidorov VA, Sadykov RA, Mel'nik NN, Gierlotka S, Presz A. Synthesis of Carbonado-Like Polycrystalline Diamond in the $\mathrm{B}_{4} \mathrm{C}-\mathrm{Graph}-$ ite System. Diamond and Related Materials. 2005; 14(3-7):437-440; 2005.

7. Elyutin VP, Poliakov VP, Fedoseev DV, Loladze NT. Effect of Amorphous Carbon on the Nucleation and Growth of Diamond Crystals. Sov. Phys. Dokl. 1987; 32(12):1013-1014.

8. Tetsuo I, Kurio A, Sakamoto S, Inoue T, Sumiya H, Funakoshi K. Formation of pure polycrystalline diamond by direct conversion of graphite at high pressure and high temperature. Physics of the Earth and Planetary Interiors. 2004; 143-144:593-600.

9. Eliutin AV, Laptev AI, Manukhin AV. "Synthesis of Carbonado Polycrystalline Diamonds from Pyrographite". Report from the Russian Academy of Science. 2001; 378(6):1-6.

10. Koltchemanov NA, Nozhkina AV, Laptev AI, Koltchemanov DN. Kinetiks of polycrystalline diamond formation. Journal of Superhard Materials. 2003; 25(2):26-32, 2003.

11. Novikov NV, Levitas VI, Leshchuk AA. Numerical Simulation of Material Stability Zones in the Working Volumes of High Pressure Equipment, Journal of Superhard Materials. 1984; 6(4):3-8.

12. Levitas VI, Idesman AV, Leshchuk AA, Polotnyak SB. Numerical modeling of thermomechanical processes in high pressure apparatus applied for superhard materials synthesis. In: Naukova Dumka, Kiev ed. High Pressure Science and Technology: Proc. Xith AIRAPT Int. Conf. 1989; 4:38-40.

13. Semionova-Tian-Chansckaia AS, Ovchinnikov AA, Microhardness Distribution in the Carbonado Body, Diamonds and Superhard Materials (in Russian). 1977; 7:1-2. 
